\title{
Partonic effects on higher-order anisotropic flows in relativistic heavy-ion collisions
}

\author{
Lie-Wen Chen, ${ }^{1, *}$ C. M. Ko, ${ }^{1}$ and Zi-Wei Lin ${ }^{2}$ \\ ${ }^{1}$ Cyclotron Institute and Physics Department, Texas A\&M University, College Station, Texas 77843-3366, USA \\ ${ }^{2}$ Physics Department, Ohio State University, Columbus, Ohio 43210, USA
}

(Received 5 January 2004; published 15 March 2004)

\begin{abstract}
Higher-order anisotropic flows $v_{4}$ and $v_{6}$ of charged hadrons in heavy-ion collisions at the Relativistic Heavy Ion Collider are studied in a multiphase transport model that has previously been used successfully for describing the elliptic flow $v_{2}$ of identified hadrons in these collisions. We find that the same parton scattering cross section of about $10 \mathrm{mb}$ used in explaining the measured $v_{2}$ of charged hadrons can also reproduce the recent data on their $v_{4}$ and $v_{6}$ from $\mathrm{Au}+\mathrm{Au}$ collisions at $\sqrt{s}=200 \mathrm{~A} \mathrm{GeV}$. It is further found that $v_{4}$ is a more sensitive probe of the initial partonic dynamics in these collisions than $v_{2}$. Moreover, higher-order parton anisotropic flows are non-negligible and satisfy the scaling relation $v_{n, q}\left(p_{T}\right) \sim v_{2, q}^{n / 2}\left(p_{T}\right)$, which leads naturally to the observed similar scaling relation among hadron anisotropic flows when the coalescence model is used to describe hadron production from the partonic matter.
\end{abstract}

DOI: 10.1103/PhysRevC.69.031901

PACS number(s): 25.75.Ld, 24.10.Lx

There have been extensive studies on the azimuthal anisotropy of hadron momentum distributions in the transverse plane perpendicular to the beam direction, particularly the lower-order elliptic flow $v_{2}$, in heavy-ion collisions at various energies [1]. The hadron transverse momentum anisotropy is generated from the pressure anisotropy in the initial compressed matter formed in noncentral heavy-ion collisions $[2,3]$ and is thus sensitive to the properties of produced matter. This sensitivity not only exists in the larger elliptic flow [4-9] but also in the smaller higher-order anisotropic flows [10-12]. Recently, higher-order anisotropic flows $v_{4}, v_{6}$, and $v_{8}$ in heavy-ion collisions at the Relativistic Heavy Ion Collider (RHIC) were studied in more detail using the hydrodynamical model [13], and it was suggested that $v_{4}$ could also provide important information on the collision dynamics during the early stage. Although the hadron anisotropic flows become smaller with increasing orders, recent experimental results from STAR Collaboration [14] have demonstrated that they are still measurable.

In the present work, the anisotropic flows $v_{2}, v_{4}$, and $v_{6}$ of both partons and hadrons in heavy-ion collisions at RHIC are studied in a multiphase transport (AMPT) model that includes initial partonic and final hadronic interactions $[15,16]$. Using the version with string melting, i.e., allowing hadrons that are expected to be formed from initial strings to convert to their valence quarks and antiquarks [17-19], we find that the measured data from $\mathrm{Au}+\mathrm{Au}$ collisions at $\sqrt{s}$ $=200 \mathrm{~A} \mathrm{GeV}$ can be approximately reproduced if a parton scattering cross section of about $10 \mathrm{mb}$ is used in the model. Our results show that the hadron $v_{4}$ is indeed a more sensitive probe of the initial partonic dynamics in relativistic heavy-ion collisions than $v_{2}$. We also find that higher-order parton anisotropic flows are non-negligible and satisfy the scaling relation $v_{n, q}\left(p_{T}\right) \sim v_{2, q}^{n / 2}\left(p_{T}\right)$, which then leads to a similar scaling relation among hadron anisotropic flows

\footnotetext{
*On leave from Department of Physics, Shanghai Jiao Tong University, Shanghai 200030, China.
}

when hadron production from the partonic matter is described by the quark coalescence model.

The AMPT model $[15,16,20,21]$ is a hybrid model that uses minijet partons from hard processes and strings from soft processes in the heavy ion jet interaction generator (HIJING) model [22] as the initial conditions for modeling heavy-ion collisions at ultrarelativistic energies. Since the initial energy density in $\mathrm{Au}+\mathrm{Au}$ collisions at RHIC is much larger than the critical energy density at which the hadronic matter to quark-gluon plasma transition would occur [20,23], we shall use the version which allows the melting of initial excited strings into partons [17]. In this version, hadrons, which would have been produced from the HIJING model, are converted instead to valence quarks and/or antiquarks [24]. Interactions among these partons are described by the Zhang's parton cascade (ZPC) model [25]. At present, this model includes only parton-parton elastic scatterings with the in-medium cross section

$$
\frac{d \sigma_{p}}{d t}=\frac{9 \pi \alpha_{s}^{2}}{2}\left(1+\frac{\mu^{2}}{s}\right) \frac{1}{\left(t-\mu^{2}\right)^{2}}
$$

where $\alpha_{s}=0.47$ is the strong coupling constant, and $s$ and $t$ are the usual Mandelstam variables. The effective screening mass $\mu$ depends on the temperature and density of the partonic matter but is taken as a parameter in ZPC for fixing the magnitude and angular distribution of parton scattering cross section. Since there are no inelastic scatterings, only quarks and antiquarks from the melted strings are present in the partonic matter. As described in Ref. [17], the transition from the partonic matter to the hadronic matter is achieved using a simple coalescence model, which combines two nearest quark and antiquark into mesons and three nearest quarks or antiquarks into baryons or antibaryons that are close to the invariant mass of these partons. The present coalescence model is thus somewhat different from the ones recently studied extensively [26-28], which are used only for producing hadrons with intermediate transverse momenta. The final-state 
hadronic scatterings are then modeled by a relativistic transport (ART) model [29]. Using parton scattering cross sections of 6-10 mb, the extended AMPT model is able to reproduce both the centrality and transverse momentum (below $2 \mathrm{GeV} / c$ ) dependence of the elliptic flow [17] and pion interferometry [18] measured in $\mathrm{Au}+\mathrm{Au}$ collisions at $\sqrt{s}=130 \mathrm{~A} \mathrm{GeV}$ at RHIC $[27,28]$. It has also been used for studying the kaon interferometry in these collisions [32].

The anisotropic flows $v_{n}$ of particles are the Fourier coefficients in the decomposition of their transverse momentum spectra in the azimuthal angle $\phi$ with respect to the reaction plane [30], i.e.,

$$
E \frac{d^{3} N}{d p^{3}}=\frac{1}{2 \pi} \frac{d N}{p_{T} d p_{T} d y}\left[1+\sum_{n=1}^{\infty} 2 v_{n}\left(p_{T}, y\right) \cos (n \phi)\right] .
$$

Because of the symmetry $\phi \leftrightarrow-\phi$ in the collision geometry, no sine terms appear in the above expansion. For particles at midrapidity in collisions with equal mass nuclei, which will be considered in the present study, anisotropic flows of odd orders vanish as a result of the symmetry $\phi \leftrightarrow \phi+\pi$. The anisotropic flows generally depend on the particle transverse momentum and rapidity, and for a given rapidity the anisotropic flows at transverse momentum $p_{T}$ can be evaluated from

$$
v_{n}\left(p_{T}\right)=\langle\cos (n \phi)\rangle,
$$

where $\langle\cdots\rangle$ denotes average over the azimuthal distribution of particles with transverse momentum $p_{T}$. The anisotropic flows $v_{n}$ can further be expressed in terms of following single-particle averages:

$$
\begin{gathered}
v_{2}\left(p_{T}\right)=\left\langle\frac{p_{x}^{2}-p_{y}^{2}}{p_{T}^{2}}\right\rangle, \\
v_{4}\left(p_{T}\right)=\left\langle\frac{p_{x}^{4}-6 p_{x}^{2} p_{y}^{2}+p_{y}^{4}}{p_{T}^{4}}\right\rangle, \\
v_{6}\left(p_{T}\right)=\left\langle\frac{p_{x}^{6}-15 p_{x}^{4} p_{y}^{2}+15 p_{x}^{2} p_{y}^{4}-p_{y}^{6}}{p_{T}^{6}}\right\rangle, \\
v_{8}\left(p_{T}\right)=\left\langle\frac{p_{x}^{8}-28 p_{x}^{6} p_{y}^{2}+70 p_{x}^{4} p_{y}^{4}-28 p_{x}^{2} p_{y}^{6}+p_{y}^{8}}{p_{T}^{8}}\right\rangle,
\end{gathered}
$$

where $p_{x}$ and $p_{y}$ are, respectively, projections of particle momentum in and perpendicular to the reaction plane.

Since the AMPT model also provides information on the spatial anisotropy of colliding matter, which is responsible for generating the momentum anisotropic flows, it is of interest to introduce the spatial anisotropic coefficient $s_{n}$ by expressions similar to those for the anisotropic flows $v_{n}$ but in terms of the spatial distributions of particles in the transverse plane.

Using the AMPT model in the string melting scenario with parton scattering cross sections $\sigma_{p}=3$ and $10 \mathrm{mb}$, we have studied the time evolutions of $s_{n}$ and $v_{n}$ of partons in midrapidity from $\mathrm{Au}+\mathrm{Au}$ collisions at $\sqrt{s}=200 \mathrm{~A} \mathrm{GeV}$ and

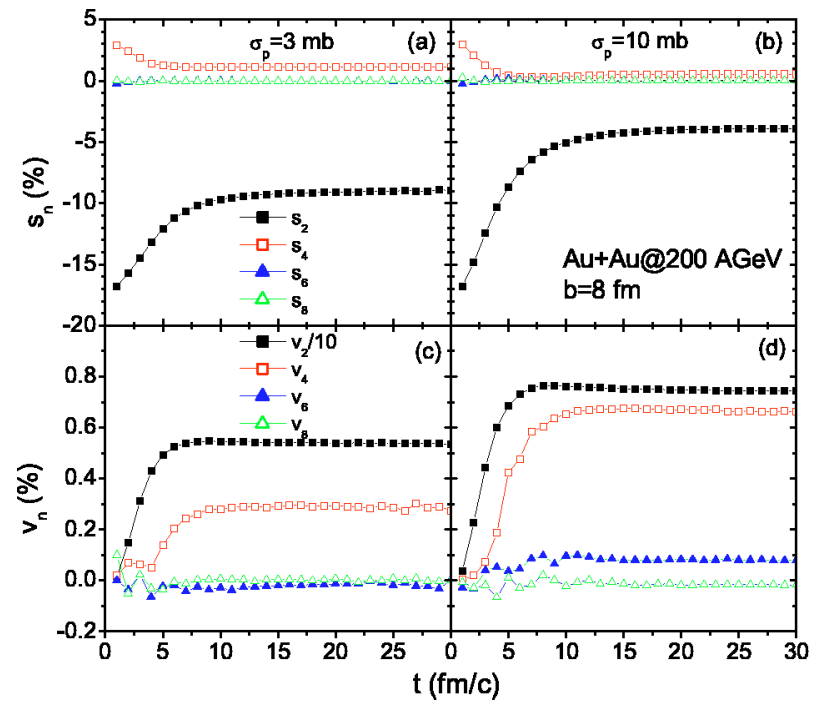

FIG. 1. (Color online) Time evolutions of spatial anisotropic coefficients $s_{n}$ and anisotropic flows $v_{n}$ of partons in midrapidity from $\mathrm{Au}+\mathrm{Au}$ collisions at $\sqrt{s}=200 \mathrm{~A} \mathrm{GeV}$ and $b=8 \mathrm{fm}$ for parton scattering cross sections $\sigma_{p}=3 \mathrm{mb}$ (left panels) and $\sigma_{p}=10 \mathrm{mb}$ (right panels).

$b=8 \mathrm{fm}$, and they are shown in Fig. 1. The spatial anisotropic coefficients $s_{n}$ at a given time are evaluated using parton positions at their last interactions before this time as in Ref. [17]. In this way, final values of $s_{n}$ are given by the parton spatial distributions at freeze-out. Similarly, final values of $v_{n}$ are determined by the parton momentum distributions at freeze-out when they are converted to hadrons by coalescence. It is seen from Figs. 1(a) and 1(b) that the spatial elliptic deformation $s_{2}$ is initially large and decreases with time. It reaches the saturation value at about $10-15 \mathrm{fm} / \mathrm{c}$ for both parton scattering cross sections. The same behavior is observed for the fourth-order spatial anisotropic coefficient $s_{4}$ except that it reaches the saturation value earlier at about $5 \mathrm{fm} / c$. The higher-order spatial anisotropic coefficients $s_{6}$ and $s_{8}$ are essentially zero. The nonzero spatial anisotropic coefficients $s_{n}$ imply that the parton spatial distribution is nonspherical at freeze-out. We note that the larger parton scattering cross section leads to a smaller spatial anisotropy at freeze-out.

The time evolutions of the momentum anisotropic flows $v_{n}$ of partons are shown in Figs. 1(c) and 1(d) for the two parton scattering cross sections of 3 and $10 \mathrm{mb}$, respectively. The elliptic flow $v_{2}$ is seen to saturate earlier in the collisions, i.e., at about $7 \mathrm{fm} / c$. This is similar for both parton scattering cross sections, except that the larger one leads to a larger elliptic flow. As to $v_{4}$ of partons, its value reaches the final value at a later time than that for $v_{2}$, i.e., at about $10 \mathrm{fm} / c$, which may be due to the fact that the parton $v_{2}$ also affects its $v_{4}$. For other higher-order anisotropic flows, $v_{6}$ of partons is very small, having a magnitude less than $0.1 \%$ for both parton scattering cross sections, while $v_{8}$ of partons is essentially zero. An interesting result predicted by the AMPT model is that the parton $v_{4}$ is more sensitive to the parton cross section than $v_{2}$. For instance, the final values of $v_{2}$ and $v_{4}$ of partons are about $5.4 \%$ and $0.29 \%$, respectively, for 


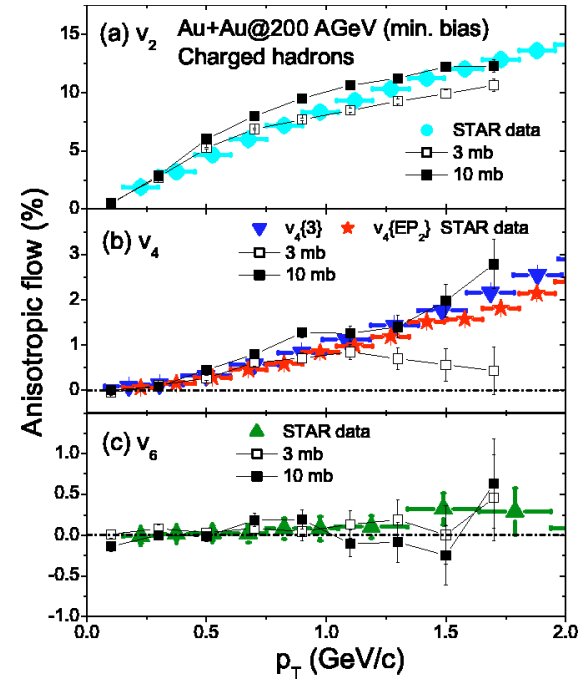

FIG. 2. (Color online) Anisotropic flows $v_{2}$ (a), $v_{4}$ (b), and $v_{6}$ (c) of charged hadrons in the pseudorapidity range $|\eta|<1.2$ from minimum bias $\mathrm{Au}+\mathrm{Au}$ collisions at $\sqrt{s}=200 \mathrm{~A} \mathrm{GeV}$ as functions of transverse momentum $p_{T}$ for parton scattering cross sections $\sigma_{p}$ $=3$ (open squares) and 10 (solid squares) $\mathrm{mb}$. The experimental data are from STAR Collaboration [14].

$\sigma_{p}=3 \mathrm{mb}$, and they are increased to about $7.5 \%$ and $0.67 \%$, respectively, for $\sigma_{p}=10 \mathrm{mb}$. Therefore, $v_{4}$ of partons is enhanced by a factor of about 2.3 while their $v_{2}$ is only enhanced by a factor of about 1.4 , when the parton cross section is increased by a factor of about 3 .

The anisotropic flows of partons are transferred to those of hadrons when the latter are formed from the coalescence of quarks and/or antiquarks. Although scatterings among hadrons are included in the AMPT model, they do not affect much the hadron anisotropy flows as a result of the small spatial anisotropy and pressure in the hadronic matter [17]. In Fig. 2, we show the final anisotropic flows $v_{n}$ of charged hadrons in the pseudorapidity range $|\eta|<1.2$ in minimum bias $\mathrm{Au}+\mathrm{Au}$ collisions at $\sqrt{s}=200 \mathrm{~A} \mathrm{GeV}$ as functions of transverse momentum $p_{T}$ for parton scattering cross sections $\sigma_{p}=3$ and $10 \mathrm{mb}$. Also shown in Fig. 2 are recent experimental data from STAR collaboration [14]. It is seen that the parton scattering cross section $\sigma_{p}=3 \mathrm{mb}$ underestimates the data at higher $p_{T}(>1 \mathrm{GeV} / c)$, while $\sigma_{p}=10 \mathrm{mb}$ seems to give a better fit to the data. The values of hadronic $v_{6}$ are in agreement with the data within error bars, although they are essentially zero. Again, $v_{4}$ of charged hadrons exhibits a stronger sensitivity to the parton cross section than their $v_{2}$, especially at higher $p_{T}$. Therefore, $v_{4}$ of hadrons provides a more sensitive probe of the initial partonic dynamics in relativistic heavy-ion collisions than their $v_{2}$.

We note that the parton scattering cross section used in the present study to reproduce the measured hadron elliptic flow is much smaller than that needed in Ref. [34] based only on the parton cascade model. This is due to the fact that in Ref. [34] the measured hadron rapidity distribution and flow were compared to those of partons. Since the number of partons in our model is about twice that in Ref. [34], we obtain a larger effect on parton elliptic flow from parton scatterings with the same cross section. Furthermore, the el-

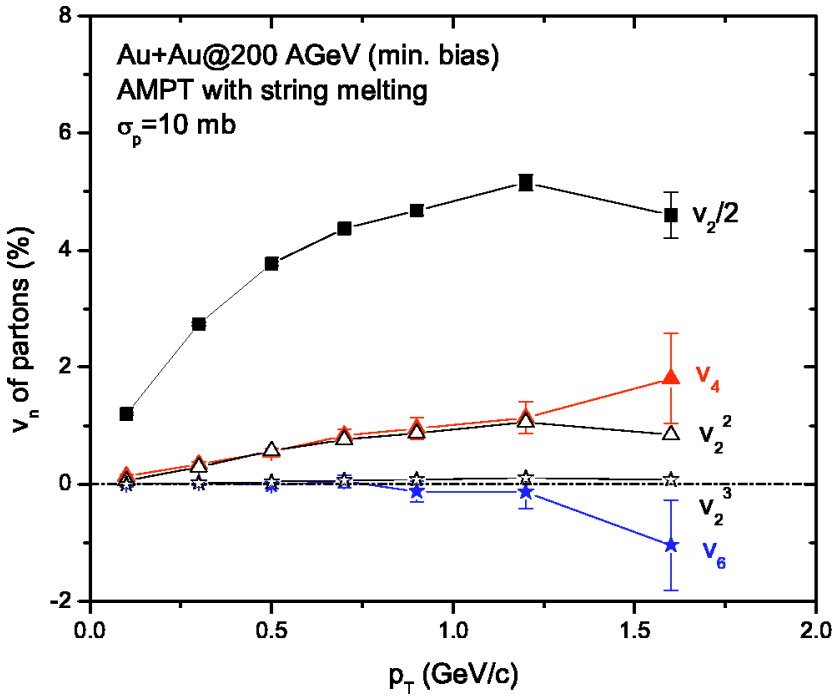

FIG. 3. (Color online) Transverse momentum dependence of midrapidity parton anisotropic flows $v_{2}, v_{4}$, and $v_{6}$ from minimum bias events for $\mathrm{Au}+\mathrm{Au}$ at $\sqrt{s}=200 \mathrm{~A} \mathrm{GeV}$ with parton scattering cross section $10 \mathrm{mb}$. Also plotted are $v_{2}^{2}$ (open triangles) and $v_{2}^{3}$ (open stars).

liptic flow of hadrons that are produced from parton coalescence is larger than that of partons if the increase of the parton elliptic flow with transverse momentum is less than linear, which is indeed the case as shown below in Fig. 3.

The experimental data indicate that there is a scaling relation among hadron anisotropic flows, i.e., $v_{n}\left(p_{T}\right)$ $\sim v_{2}^{n / 2}\left(p_{T}\right)$ [14]. It has been shown by Kolb [35] that such scaling relation follows naturally from a naive quark coalescence model [36] that only allows quarks with equal momentum to form a hadron. Denoting the meson anisotropic flows by $v_{n, M}\left(p_{T}\right)$, he found that $v_{4, M}\left(p_{T}\right)=(1 / 4) v_{2, M}^{2}\left(p_{T}\right)$ if quarks have no higher-order anisotropic flows. Since mesons dominate the yield of charged particles in experimental data, the smaller scaling factor of $1 / 4$ than the empirical value of about 1 indicates that higher-order quark anisotropic flows cannot be neglected. Including the latter contribution, one can show that

$$
\begin{gathered}
\frac{v_{4, M}\left(2 p_{T}\right)}{v_{2, M}^{2}\left(2 p_{T}\right)} \approx \frac{1}{4}+\frac{1}{2} \frac{v_{4, q}\left(p_{T}\right)}{v_{2, q}^{2}\left(p_{T}\right)}, \\
\frac{v_{6, M}\left(2 p_{T}\right)}{v_{2, M}^{3}\left(2 p_{T}\right)} \approx \frac{1}{4}\left(\frac{v_{4, q}\left(p_{T}\right)}{v_{2, q}^{2}\left(p_{T}\right)}+\frac{v_{6, q}\left(p_{T}\right)}{v_{2, q}^{3}\left(p_{T}\right)}\right),
\end{gathered}
$$

where $v_{n, q}\left(p_{T}\right)$ denotes the quark anisotropic flows. The meson anisotropic flows thus satisfy the scaling relations if the quark anisotropic flows also satisfy such relations.

In Fig. 3, we show the $p_{T}$ dependence of the anisotropic flows $v_{2}, v_{4}$, and $v_{6}$ of midrapidity partons from minimum bias events in $\mathrm{Au}+\mathrm{Au}$ collisions at $\sqrt{s}=200 \mathrm{~A} \mathrm{GeV}$ for a parton scattering cross section of $10 \mathrm{mb}$. Also shown in Fig. 3 are $v_{2}^{2}$ (open triangles) and $v_{2}^{3}$ (open stars). It is seen that the parton anisotropic flows from the AMPT model indeed sat- 
isfy the scaling relation $v_{n, q}\left(p_{T}\right) \sim v_{2, q}^{n / 2}\left(p_{T}\right)$. With a parton scaling factor of about 1, we obtain the following scaling relations among meson anisotropic flows:

$$
\frac{v_{4, M}\left(p_{T}\right)}{v_{2, M}^{2}\left(p_{T}\right)} \approx \frac{3}{4}, \quad \frac{v_{6, M}\left(p_{T}\right)}{v_{2, M}^{3}\left(p_{T}\right)} \approx \frac{1}{2} .
$$

The resulting hadron scaling factors of $3 / 4$ and $1 / 2$ are, however, smaller than the one extracted from measured anisotropic flows of charged hadrons. Since the naive quark coalescence model does not allow hadron formation from quarks with different momenta as in more realistic quark coalescence models [26-28], it is not expected to give a quantitative description of the experimental observation. Such effects are, nevertheless, included in the AMPT model, which have been shown in Fig. 2 to reproduce the measured hadron anisotropic flows.

In summary, using AMPT model in the string melting scenario, we have studied the anisotropic flows $v_{2}, v_{4}$, and $v_{6}$ of both partons and hadrons in heavy-ion collisions at RHIC. We find that the anisotropic flows are developed early in the collision when the matter is in the partonic stage and has appreciable spatial anisotropy. With a parton scattering cross section of about $10 \mathrm{mb}$, recent data on hadron isotropic flows from $\mathrm{Au}+\mathrm{Au}$ collisions at $\sqrt{s}=200 \mathrm{~A} \mathrm{GeV}$ can be approximately reproduced. Our results further show that $v_{4}$ is a more sensitive probe of the initial partonic dynamics in these collisions than $v_{2}$, and that higher-order parton anisotropic flows are non-negligible and satisfy the scaling relation $v_{n, q}\left(p_{T}\right)$ $\sim v_{2, q}^{n / 2}\left(p_{T}\right)$. Using the quark coalescence model, we then show that the parton scaling relation leads naturally to the observed similar scaling relation among hadron anisotropic flows. Studies of higher-order anisotropic flows in relativistic heavy-ion collisions are thus expected to provide useful information on the collision dynamics and the properties of the hot dense matter during the initial stage.

We thank Vincenzo Greco, Peter Kolb, and Ralf Rapp as well as Art Poskanzer and $\mathrm{Nu} \mathrm{Xu}$ for useful discussions. This paper was based on work supported by the U.S. National Science Foundation under Grant No. PHY-0098805 and the Welch Foundation under Grant No. A-1358. L.W.C. was also supported by the National Natural Science Foundation of China under Grant No. 10105008.
[1] W. Reisdorf and H. G. Ritter, Annu. Rev. Nucl. Part. Sci. 47, 663 (1997).

[2] J. Barrette et al., E877 Collaboration, Phys. Rev. Lett. 73, 2532 (1994).

[3] H. Appelshauser et al., NA49 Collaboration, Phys. Rev. Lett. 80, 4136 (1998).

[4] J. Y. Ollitrault, Phys. Rev. D 46, 229 (1992).

[5] H. Sorge, Phys. Lett. B 402, 251 (1997); Phys. Rev. Lett. 78, 2309 (1997); 82, 2048 (1999).

[6] P. Danielewicz, R. A. Lacey, P. B. Gossiaux, C. Pinkenburg, P. Chung, J. M. Alexander, and R. L. McGrath, Phys. Rev. Lett. 81, 2438 (1998).

[7] B. Zhang, M. Gyulassy, and C. M. Ko, Phys. Lett. B 455, 45 (1999).

[8] Y. Zheng, C. M. Ko, B. A. Li, and B. Zhang, Phys. Rev. Lett. 83, 2534 (1999).

[9] S. A. Voloshin, Nucl. Phys. A715, 379c (2003).

[10] P. F. Kolb, J. Sollfrank, and U. Heinz, Phys. Lett. B 459, 667 (1999).

[11] D. Teaney and E. V. Shuryak, Phys. Rev. Lett. 83, 4951 (1999).

[12] P. F. Kolb, J. Sollfrank, and U. Heinz, Phys. Rev. C 62, 054909 (2000).

[13] P. F. Kolb, Phys. Rev. C 68, 031902(R) (2003).

[14] J. Adams et al., STAR Collaboration, Phys. Rev. Lett. 92, 062301 (2004).

[15] B. Zhang, C. M. Ko, B. A. Li, and Z. W. Lin, Phys. Rev. C 61, 067901 (2000).

[16] Z. W. Lin, S. Pal, C. M. Ko, B. A. Li, and B. Zhang, Phys. Rev. C 64, 011902 (2001); Nucl. Phys. A698, 375 (2002).

[17] Z. W. Lin and C. M. Ko, Phys. Rev. C 65, 034904 (2002).

[18] Z. W. Lin, C. M. Ko, and S. Pal, Phys. Rev. Lett. 89, 152301 (2002).
[19] C. M. Ko, Z. W. Lin, and S. Pal, Heavy Ion Phys. 17, 219 (2003).

[20] B. Zhang, C. M. Ko, B. A. Li, Z. W. Lin, and B. H. Sa, Phys. Rev. C 62, 054905 (2000); B. Zhang, C. M. Ko, B. A. Li, Z. W. Lin, and S. Pal, ibid. 65, 054909 (2002).

[21] S. Pal. C. M. Ko, and Z. W. Lin, Nucl. Phys. A730, 143 (2004).

[22] X. N. Wang and M. Gyulassy, Phys. Rev. D 44, 3501 (1991).

[23] D. Kharzeev and M. Nardi, Phys. Lett. B 507, 121 (2001).

[24] T. Sjostrand, Comput. Phys. Commun. 82, 74 (1994).

[25] B. Zhang, Comput. Phys. Commun. 109, 193 (1998).

[26] V. Greco, C. M. Ko, and P. Lévai, Phys. Rev. Lett. 90, 202302 (2003); Phys. Rev. C 68, 034904 (2003).

[27] R. C. Hwa and C. B. Yang, Phys. Rev. C 67, 034902 (2003); 67, 064902 (2003).

[28] R. J. Fries, B. Müller, C. Nonaka, and S. A. Bass, Phys. Rev. Lett. 90, 202303 (2003); Phys. Rev. C 68, 044902 (2003).

[29] B. A. Li and C. M. Ko, Phys. Rev. C 52, 2037 (1995); B. A. Li, A. T. Sustich, B. Zhang, and C. M. Ko, Int. J. Mod. Phys. E 10, 267 (2001).

[30] K. H. Ackermann et al., STAR Collaboration, Phys. Rev. Lett. 86, 402 (2001).

[31] C. Adler et al., STAR Collaboration, Phys. Rev. Lett. 87, 082301 (2001).

[32] Z. W. Lin and C. M. Ko, J. Phys. G 30, S263 (2004).

[33] A. M. Poskanzer and S. A. Voloshin, Phys. Rev. C 58, 1671 (1998).

[34] D. Molnar and M. Gyulassy, Nucl. Phys. A697, 495 (2002); A703, 893 (2002).

[35] P. Kolb, Talk at the RIKEN-BNL Workshop on Collective Flow and QGP Properties, Bookhaven National Laboratory, 2003 (unpublished), http://tonic.physics.sunysb.edu/flow03

[36] D. Molnar and S. A. Voloshin, Phys. Rev. Lett. 91, 092301 (2003). 